\title{
Overview of the EU FP7-project HISTORIC
}

\author{
G. Morthier, R. Kumar \\ Department of Information Technology, Ghent University, B-9000 Gent, Belgium \\ F. Raineri, R. Raj \\ CNRS, Lab Photon \& Nanostruct, F-91460 Marcoussis, France \\ Jens Hofrichter, Nikolaos Chrysos, B.J. Offrein \\ IBM Research GmbH - IBM Research - Zurich, Säumerstrasse 4, 8803 \\ Rüschlikon/Switzerland \\ R. Zhang, J. van der Tol, O. Raz, H. Dorren \\ COBRA Research Institute, Technische Universiteit Eindhoven, 5600 MB Eindhoven, The \\ Netherlands
}

\begin{abstract}
HISTORIC aims to develop and test complex photonic integrated circuits containing a relatively large number of digital photonic elements for use in e.g. all-optical packet switching. These photonic digital units are all-optical flip-flops based on ultra compact laser diodes, such as microdisk lasers and photonic crystal lasers. These lasers are fabricated making use of the heterogeneous integration of InP membranes on top of silicon on insulator (SOI) passive optical circuits. The very small dimensions of the lasers are, at least for some approaches, possible because of the high index contrast of the InP membranes and by making use of the extreme accuracy of CMOS processing.

All-optical flip-flops based on heterogeneously integrated microdisk lasers with diameter of $7.5 \mu \mathrm{m}$ have already been demonstrated. They operate with a CW power consumption of a few $\mathrm{mW}$ and can switch in 60ps with switching energies as low as $1.8 \mathrm{fJ}$. Their operation as all-optical gate has also been demonstrated.

Work is also on-going to fabricate heterogeneously integrated photonic crystal lasers and all-optical flip-flops based on such lasers. A lot of attention is given to the electrical pumping of the membrane InP-based photonic crystal lasers and to the coupling to SOI wire waveguides. Optically pumped photonic crystal lasers coupled to SOI wires have been demonstrated already.

The all-optical flip-flops and gates will be combined into more complex photonic integrated circuits, implementing all-optical shift registers, D flip-flops, and other all-optical switching building blocks.

The possibility to integrate a large number of photonic digital units together, but also to integrate them with compact passive optical routers such as AWGs, opens new perspectives for the design of integrated optical processors or optical buffers. The project therefore also focuses on designing new architectures for such optical processing or buffer chips.
\end{abstract}

Keywords: Heterogeneous integration, digital photonics, all-optical flip-flops

\section{Introduction}

Many presently existing devices for all-optical signal processing have a very large footprint and power consumption, making it hard for all-optical signal processing to compete with opto-electronic solutions. Often several active elements (laser diodes, semiconductor optical amplifiers) or bulky non-linear fibers are required and the power consumption for simple operations like wavelength conversion or all-optical flip-flopping easily reaches several Watts. Moreover, integration on small chip areas is often not possible due to the need for isolators and relatively long delay lines [1-2].

Therefore, the HISTORIC project aimed at developing components and photonic integrated circuits allowing all-optical signal processing with much lower power consumption and 
footprint and allowing the fabrication of more complex optical circuits as photonic integrated circuits. This is done using two different approaches, one based on micodisk lasers and resonators and one based on photonic crystal lasers and resonators. The results obtained with the different approaches are described hereafter.

\section{Microdisk based devices}

The work on these devices focused in the first year a lot on obtaining good all-optical set-reset flip-flops using heterogeneously integrated single or coupled microdisk lasers. In addition, there has been activity on fabricating photonic integrated circuits containing multiplexers/demultiplexers and gates.

From earlier theoretical work, it was known that all-optical flip-flops with ring or disk lasers can only result if the coupling between the clockwise (CW) and counter clockwise (CCW) mode is sufficiently small and if a sufficiently high internal power or photon density can be achieved in the whispering gallery mode of the disks [3].

The first microdisk lasers that we measured (fabricated as part of an earlier FP6 project, PICMOS) didn't exhibit bistable unidirectional behaviour [4]. However, using the RF-spectra obtained from the output of these lasers we were able to estimate the coupling between $\mathrm{CW}$ and CCW modes. This coupling gives a frequency split in the spectrum of the total optical field, which can easily be seen in the spectrum of e.g. the Relative Intensity Noise (RIN). More exactly, the resonance frequency itself is given by $K_{c} / \pi$, while the $6 \mathrm{~dB}$ width of the resonance is given by $K_{d} / \pi$. The figure 2.1 below shows such spectra at 3 different bias currents for a typical disk laser of the first generation. For the spectrum of Figure 2, the resonance corresponds with $\mathrm{K}_{\mathrm{c}}=610^{9} \mathrm{~s}^{-1}$ and $\mathrm{K}_{\mathrm{d}}=310^{8} \mathrm{~s}^{-1}$.

Using these data for the coupling coefficients and some good estimations for other laser parameters (such as the gain parameters), we performed numerical simulations to see how much we needed to improve specific parameters in order to obtain bistable unidirectional operation. The simulation program is basically a simple rate equation program, with 2 coupled rate equations for the $\mathrm{CW}$ and CCW complex field amplitudes and one carrier density rate equation.

We found that obtaining unidirectional operation would require a coupling between CW and CCW modes which is at least 3 to 4 times smaller than observed in the actual devices. In addition, having higher power levels in our disk lasers would also be very beneficial. In the first disks, the power seemed to saturate above current levels of $2 \mathrm{~mA}$ due to strong heating, which in turn is due to the bad thermal conductivity of the bonding layers.

We also extended the rate equation model to a model for coupled microdisk lasers and did some extensive simulations for these lasers. Our simulations of coupled microdisk lasers showed that coupled microdisk could be bistable and unidirectional for lower power levels or larger coupling between CW and CCW fields. It depends however on the phase shift corresponding with the coupling between the disks. For some phase shift values, also chaotic behaviour could be observed. However, obtaining unidirectional behaviour in coupled disk is only possible on the condition that the free running wavelengths of the two disks are sufficiently close to each other. This is a condition that is specific for coupled disks and makes it more complicated to obtain S-R flip-flops using coupled disk lasers. With disks diameters in the order of $10 \mu \mathrm{m}$, small changes in the disk diameters might already cause relatively large changes in the laser wavelength.

We therefore focused on obtaining unidirectional behaviour in single disk lasers. 


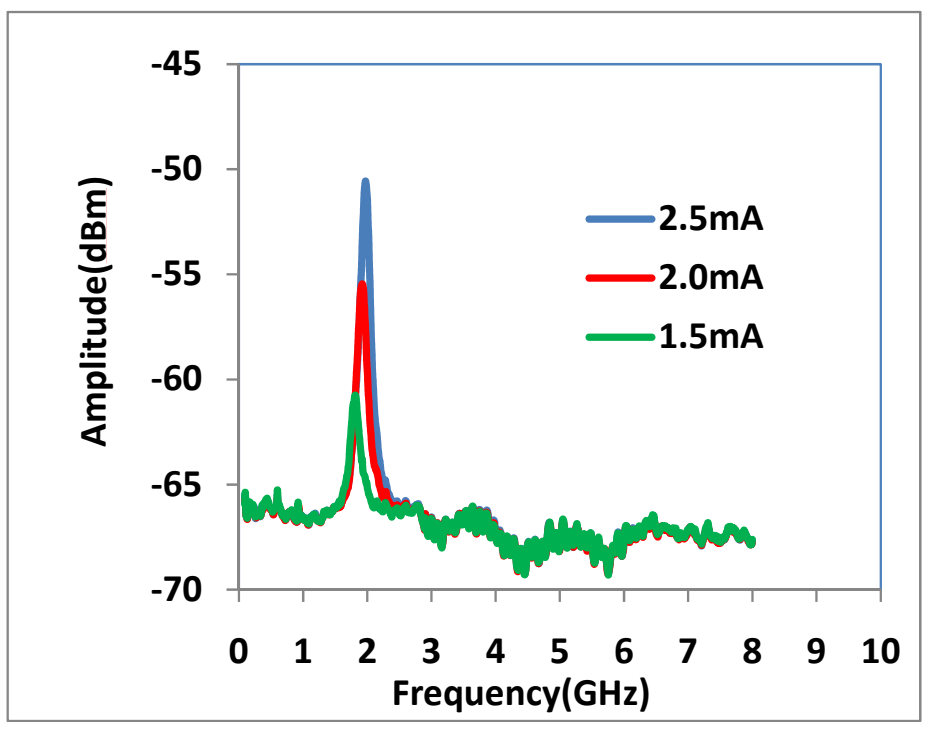

Figure 2.1. RF-spectra at different currents of a microdisk laser with diameter $10 \mu \mathrm{m}$. The resonance due to the coupling between $\mathrm{CW}$ and CCW mode is clearly visible.

Using the data extracted for the coupling coefficients and some good estimations for other laser parameters (such as the gain parameters), we found from our simulations that obtaining unidirectional operation would require a coupling between CW and CCW modes which is at least 3 to 4 times smaller. In addition, having higher power levels in our disk lasers would also be very beneficial.

For the above reasons, we tried both to obtain higher internal power in the disks and to reduce the coupling between CW and CCW modes. Obtaining higher internal power was pursued by improving the heat sinking by depositing a thick Au layer on top of the microdisk that functions as heat sink. As will be discussed later, this allowed to obtain much higher power levels.

The reduction of the coupling between CW and CCW modes was pursued in two ways. First, it was realized that the reflection from the grating couplers (estimated to be $-22 \mathrm{~dB}$ ), together with the 1-2\% coupling between disk and $\mathrm{Si}$ wire waveguides could already cause a significant coupling between CW and CCW modes and therefore new chips were designed with some of the disk lasers connected to Si wires which eventually are turned into angled waveguides. This would allow to cleave the Si chips and obtain angled facets instead of grating couplers for coupling to fiber.

Secondly, we also realized that the surface roughness of the sidewalls of the disk was too large and gave a lot of scattering. To reduce this roughness, new disks were etched at the facilities and using the recipe of the HISTORIC partner TUE. This resulted in a significant reduction of the surface roughness as is illustrated in Figure 2.2 where SEM pictures are shown from both the old disks (with large surface roughness) and the latest disks (with reduced roughness).

Measurements on the newly fabricated microdisk lasers quickly revealed that they indeed become bistable and unidirectional at relatively low current levels and this even for disk diameters as small as $7.5 \mu \mathrm{m}$. The L-I characteristics measured for a disk with $7.5 \mu \mathrm{m}$ diameter, with the power measured at both facets (i.e. for the clockwise and the counter clockwise mode) is given in Figure 2.3 (lhs). The output power is now also more than an order of magnitude higher than in previously fabricated disks. 

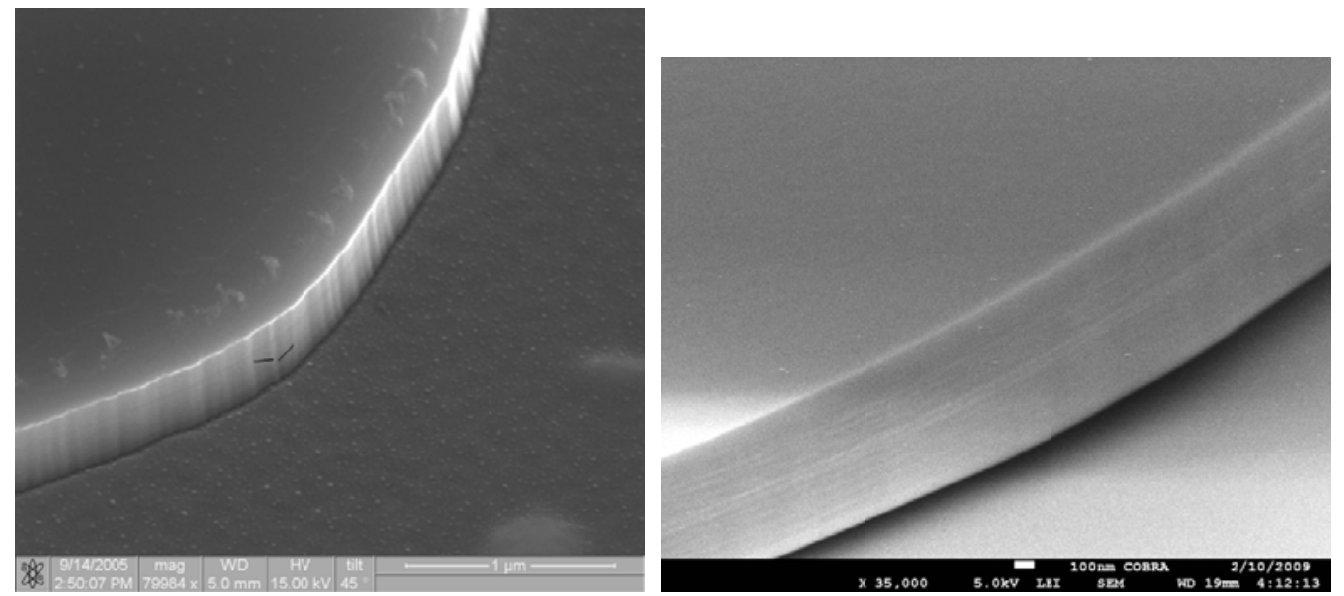

Figure 2.2. sidewall surface roughness of older microdisk lasers (left) and of the latest lasers (right).

Figure 2.3 (rhs) shows one of the switching results that have been obtained so far with one of the microdisk all-optical flip-flops. The figure shows the (amplified) output power in the CW mode vs. time. The set pulses can't be distinguished from the laser power itself though. The switching pulses have a duration of $100 \mathrm{ps}$ and an energy as small as $1.8 \mathrm{fJ}$. Switching times as small as 60 ps have been obtained in the first experiments, but more experiments are required to optimise the switching.
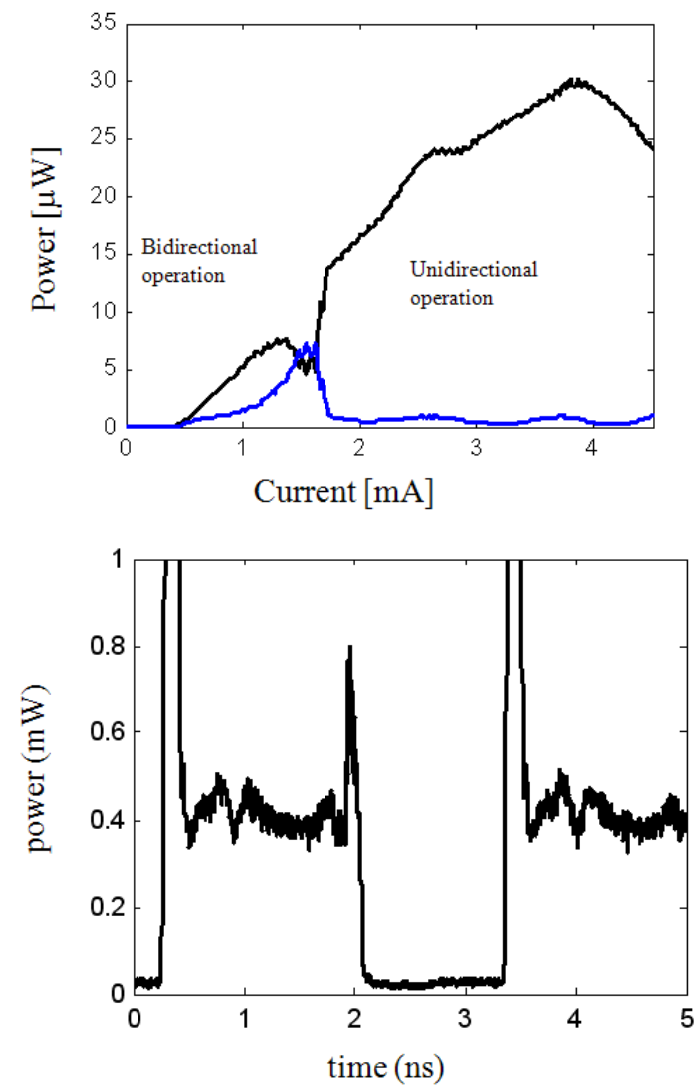

Figure 2.3. (left) L-I characteristic of a heterogeneously integrated microdisk laser with diameter $7.5 \mu \mathrm{m}$. The blue line is the power emitted from the l.h.s. (the CW mode) and the 
black line is the power emitted from the r.h.s. (the CCW mode); (right) Time trace of the laser output power showing the setting and resetting.

Another attractive function for MDLs is wavelength conversion [6-7] which is a key functionality in photonic networks. Recently we have been experimenting with the latest generation of MDLs in order to prove their applicability for low power and small foot-print converters. Many advances in the design and fabrication of MDLs have been gained through the HISTORIC project. For the devices used for the wavelength conversion experiments, one striking feature was the very low threshold current required to achieve lasing. In Figure 2.4 we show the L-I curve for two such MDLs with a threshold between 0.1-0.2mAmps (and the MDL spectra in the inset at a current of $150 \mu \mathrm{Amps}$ ).

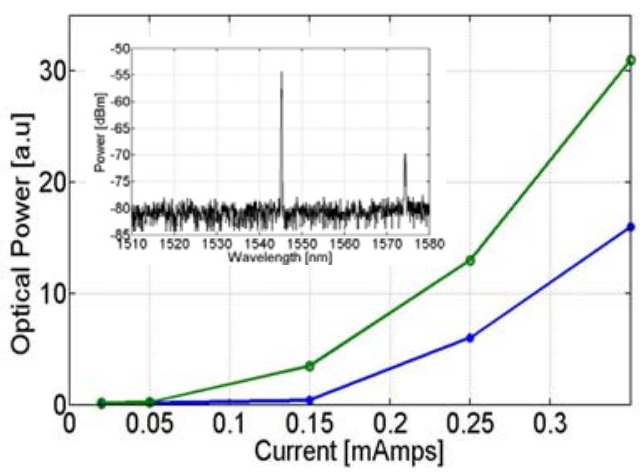

In Figure 2.4, we also give the experimental set-up used for this demonstration. The pump signal is a tunable laser source tuned to the non-dominant lasing mode of the MDL and modulated by a PRBS generator at 2.5,10 and 20Gb/s using an MZM. From the other side of the disc a 2nd laser (seed) is tuned to the dominant lasing mode under free running operation. Power levels are assumed in the silicon waveguide based on power in the input to the fiber lens and assuming $8 \mathrm{~dB}$ coupling losses. The free running laser light at the circulator's output is filtered, amplified using an EDFA and filtered again to remove excessive ASE noise before detection at the appropriate receivers (APD for $2.5 \mathrm{~Gb} / \mathrm{s} ; 10 \mathrm{~Gb} / \mathrm{s}$, PIN for 20Gb/s). The device was a $7.5 \mu \mathrm{m}$ diameter InP disk laser lithographically etched in a 580nm thick membrane bonded on top of an SOI waveguide circuit. The disc was operated at $15^{\circ} \mathrm{C}$ and biased at 2.5 $\mathrm{mA}$ (3volt) to give $\sim 10 \mu \mathrm{W}$ of power in the fiber. Gratings at both ends of the silicon waveguides allowed for coupling of light to the chip.

Fig. 2.5 shows the MDLs output spectra under three operation conditions. On the top, free running operation is shown, indicating $>20 \mathrm{~dB}$ side mode suppression. Once the pump laser is turned on, the free running laser power (middle frame) drops by $9 \mathrm{~dB}$ due to the limited recovery speed of the MDL, effectively inhibiting conversion. The solution is to introduce the seed laser [6], which speeds the MDL free running mode recovery, gaining back 6dB to leave an expected $3 \mathrm{~dB}$ power gap between modulated and un-modulated spectra's (bottom frame). For different modulation speeds the reference performance of the receiver (Receiver Power vs. BER) were measured and compared to the wavelength converted signals. Curves for all measured modulation speeds are shown in Figure 2.6. For the $2.5 \mathrm{~Gb} / \mathrm{s}$ and $10 \mathrm{~Gb} / \mathrm{s}$ signals the EDFA operated with low to moderate gains yielding $0.8 \mathrm{~dB}$ and $4.5 \mathrm{~dB}$ penalty respectively, mainly depending on available OSNR. 
For $20 \mathrm{~Gb} / \mathrm{s}$ rate, the receiver used had twice the required bandwidth (43Gb/s module), doubling the noise contribution, and much lower sensitivity forcing higher gain from the EDFA and further degrading the noise figure to leave a distinct error floor. Power was kept below $-3 \mathrm{dBm}$ to avoid damaging the receiver resulting in BER under $10^{-3}$ but no error free operation.
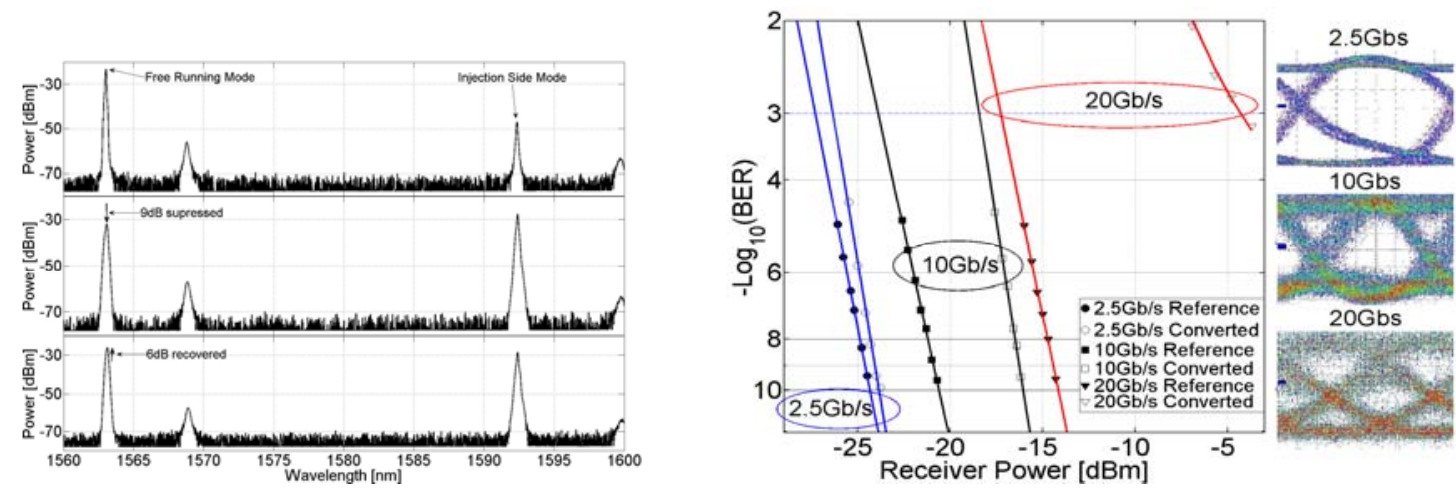

\section{Photonic Crystal laser based devices}

We also aim at obtaining new generations of all optical flip-flops with improved performance with respect to the micro-disk based approach in terms of size, speed and power consumption. We had set ourselves two paths to be explored: one based on two-dimensional photonic crystal (2DPC) lasers based on optical pumping, for which mainly LPN is responsible, and another based on photonic crystal lasers with direct electrical injection, for which mainly TUe is responsible. Both of these approaches are very exploratory and as such require intense effort in numerical studies and technological development.

We describe in Section 3.1 our effort on the integration of two material levels devoted to different functionalities: SOI photonic wires and InP-based photonic crystals, using adhesive bonding. We describe briefly the fabrication procedure beginning with the method used to bond the InP and SOI layers. Then we disscuss the processing procedures for patterning in the InP layer aligned with the SOI waveguides ending finally with a report on the optical measurements of the device emission.

In Section 3.2 we report the preliminary work done towards Photonic crystal structure with direct electrical injection.

\subsection{Heterogeneously integrated III-V PhC optically pumped lasers onto SOI wavequides}

The sample that we fabricated is schematically represented in Figure 3.1. It consists of a twooptical-level structure: the lower level is composed of SOI narrow waveguides (width 300nm - height 220nm) where the light propagates passively, i.e. with neither absorption nor nonlinear interaction with the matter and in specific areas, an upper level is added. The latter is a $250 \mathrm{~nm}$ thick InP-based membrane encompassing $4 \mathrm{InGaAsP} / \mathrm{InGaAs}$ quantum wells (QWs) emitting drilled to give a two dimensional photonic crystal structure (2D PhC).

\subsubsection{Bonding}


The InP material is grown at LPN by MOCVD, and contains four InGaAs/InGaAsP Quantum Wells emitting at a peak photoluminescence wavelength of $1.53 \mu \mathrm{m}$. The SOI waveguides are patterned by 193nm DUV lithography on a CMOS line at IMEC, Belgium. These are then etched using a $\mathrm{ClHBr}$ Reactive Ion Etch. Different widths of ridge waveguide are produced to allow phase matching to structures with various effective indices. The $220 \mathrm{~nm}$ Si layer is entirely etched, leaving a 220*500 waveguide with a $2 \mu \mathrm{m}$ BuriedOXide (BOX). Alignment marks for subsequent processing are defined in the Si on the same mask layer and relative to the waveguide structures.

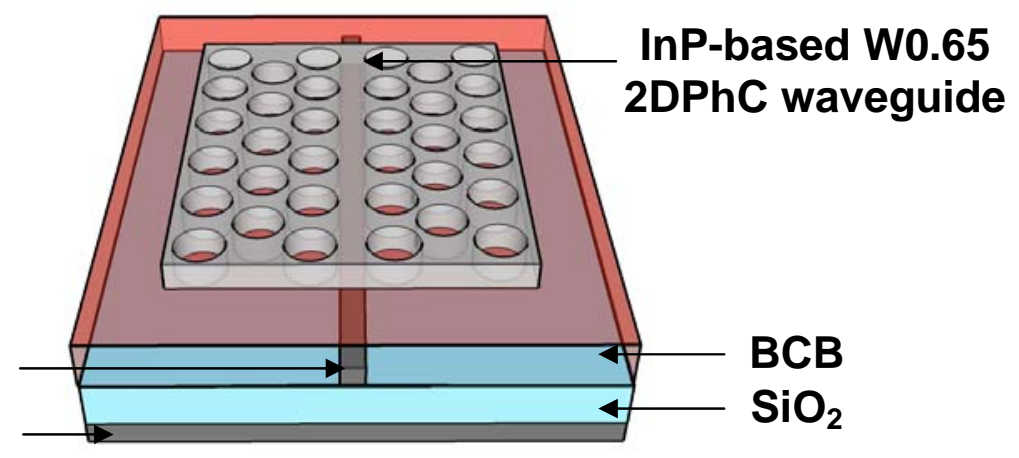

Figure 3.1: Schematic representation of the sample

A $1.4 \times 1 \mathrm{~cm}^{2}$ SOI die is diced from an 8 " wafer and a roughly $5 \mathrm{~mm}^{2}$ die of InP is cleaved. To achieve a high quality bonded interface, our first requirement is two clean surfaces. The BCB is then diluted in an appropriate ratio of mesitylene in order to obtain the chosen spun film thickness. After application of an adhesion promoter (AP3000) the BCB is spun (5000RPM, $30 \mathrm{~s})$ on to the SOI, degassing takes place at $80^{\circ} \mathrm{C}$ for several minutes preventing the appearance of bubbles, that introduce defects in the final structure. Finally the InP material is gently placed over the site of the waveguides, whilst the sample is heated to $150^{\circ} \mathrm{C}$. In order to polymerise the BCB and finalise the bonding a hard bake is performed in a nitrogen atmosphere for 2 hours at $350^{\circ} \mathrm{C}$. Once hard baked the InP substrate is removed by $\mathrm{HCl}$ wet etch. The InGaAs etch stop is removed using $\mathrm{H}_{2} \mathrm{SO}_{4}: \mathrm{H}_{2} \mathrm{O}_{2}: \mathrm{H}_{2} \mathrm{O}$ to leave a $255 \mathrm{~nm} \mathrm{InP}$ membrane bonded to the SOI.

\subsubsection{III-V PhC patternig and Alignment to SOI waveguides}

The next step is the patterning of the photonic crystals in the bonded InP material and this requires a two stage masking process. A $300 \mathrm{~nm} \mathrm{Si}_{3} \mathrm{~N}_{4}$ mask is deposited by PECVD, to act as a hard mask. This layer also serves to protect the BCB and underlying Silicon guides in later processing steps. On top of this we spin coat and bake a 450nm PMMA mask. It is very important initially to determine the alignment marks very precisely in order to position the photonic crystal waveguides right on top of the SOI wires. To align our guides our "semiautomatic" alignment technique requires four marks, defined in the same high resolution mask layer as the Si waveguides. The abruptness of the edges of the mark is paramount to ensuring high contrast. In these thin semiconductor films, at the high electron writing energy (100keV) employed, the backscattered signal is characteristically weak and of poor contrast. To add to this difficulty, the multilayer semiconductor/dielectric stack is prone to charging, as the BCB is a particularly efficient insulating layer.

The marks allow us to correct for the rotation, shift and scaling between the Si and InP layers. The photonic crystal waveguide patterns are exposed in the PMMA along with a $2 \mu \mathrm{m}$ wide 
moat (in the same high resolution layer). This allows us to very simply discard the superfluous InP at a later processing step, avoiding the absorption that leaving this material would cause. Following development of the PMMA in a MIBK/IPA (1:3) solution, the pattern is transferred into the $\mathrm{Si}_{3} \mathrm{~N}_{4}$ mask by a Reactive Ion Etch using a $\mathrm{SF}_{6} / \mathrm{CHF}_{3}$ chemistry (gas ratio 10/14 sccm). To avoid reflow at the subsequent high temperatures the PMMA is also removed in a RIE dry etch organic cleaning process. The photonic crystal is etched into the InP layer by ICP-RIE with $\mathrm{Cl}_{2} / \mathrm{BCl}_{3} \mathrm{~N}_{2}$ chemistry. The $\mathrm{Si}_{3} \mathrm{~N}_{4}$ mask is then removed by repeating the previous RIE process. We then wish to remove the superfluous InP material. This is achieved using the alignment marks in collaboration with a negative resist (mAN 2410).

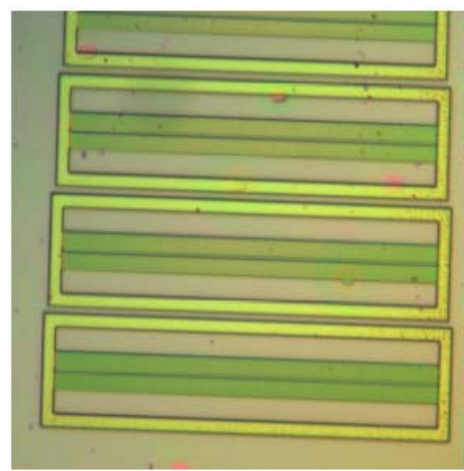

(a)

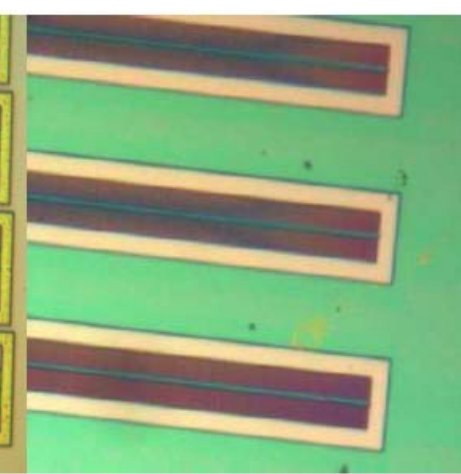

(b)

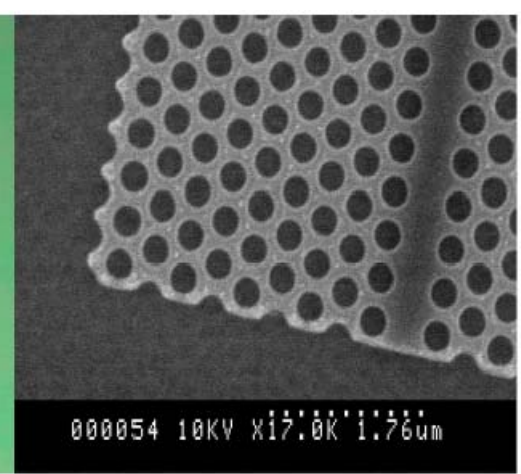

(c)

Figure 3.2: (a) Optical images showing the PhC patterning of first the soft PMMA mask (aligned with the guide below) and (b) its subsequent transfer into the $\mathrm{Si}_{3} \mathrm{~N}_{4}$ hard mask by RIE etch. (c) An SEM image reveals the island of patterned $\mathrm{Si}_{3} \mathrm{~N}_{4}$.
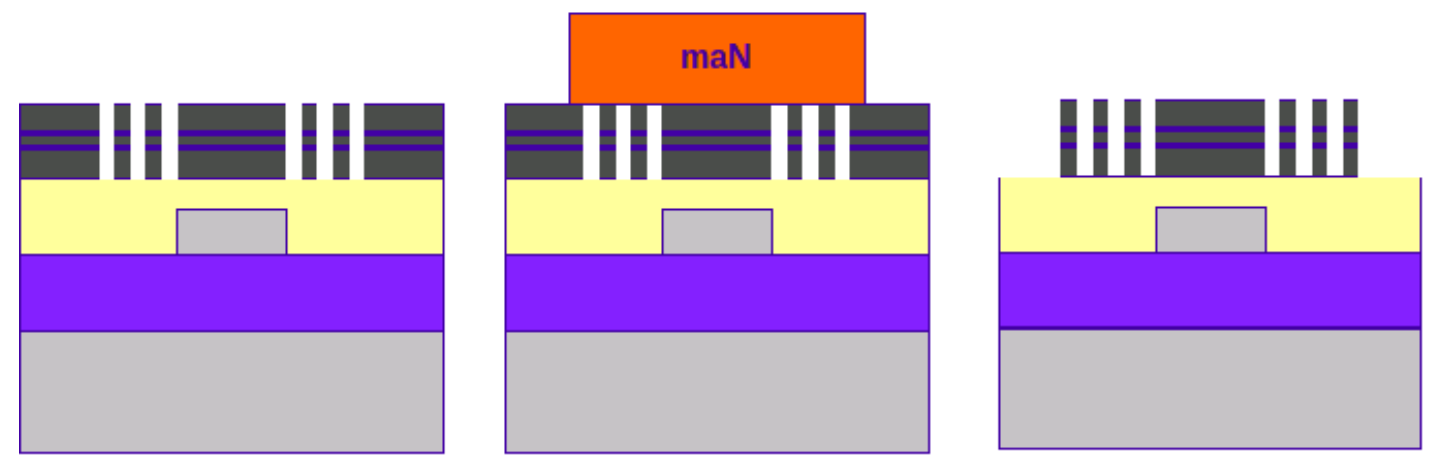

Figure 3.3: The InP layer is etched using ICP, and in the lower optical image the Si waveguides become visible. The $\mathrm{Si}_{3} \mathrm{~N}_{4}$ mask is removed and a negative resist maN is patterned to efficiently protect the PhC whilst the superfluous InP material is removed. The final structures bonded, accurately aligned and patterned on top of the Si wire waveguides.

A low resolution area, is exposed by the Leica, which is $1 \mu \mathrm{m}$ narrower than the moat. This mesa protects the underlying photonic crystal from a three step wet etching cycle using $\mathrm{HCl}$, $\mathrm{H}_{2} \mathrm{SO}_{4}: \mathrm{H}_{2} \mathrm{O}_{2}: \mathrm{H}_{2} \mathrm{O}$ (3:1:1) and once again $\mathrm{HCl}$, to remove the InP and the InGaAs/InGaAsP Quantum Wells. The entire procedure is depicted in Fig.3.4. 


\subsubsection{Alignment accuracy estimation.}

In order to give a satisfactory estimate of the alignment error between the two patterned layers, we capitalised the fact that the BCB was slightly thinned in the InP etching step to obtain reasonably contrasted SEM images of the ends of the photonic crystals and the silicon waveguide layer, beneath the residual BCB (Fig. 3.4). By fitting the waveguide position and the hole centres over a selection of guides, we can gain a statistical measure of our alignment capabilities. We determine an alignment accuracy of 30nm.

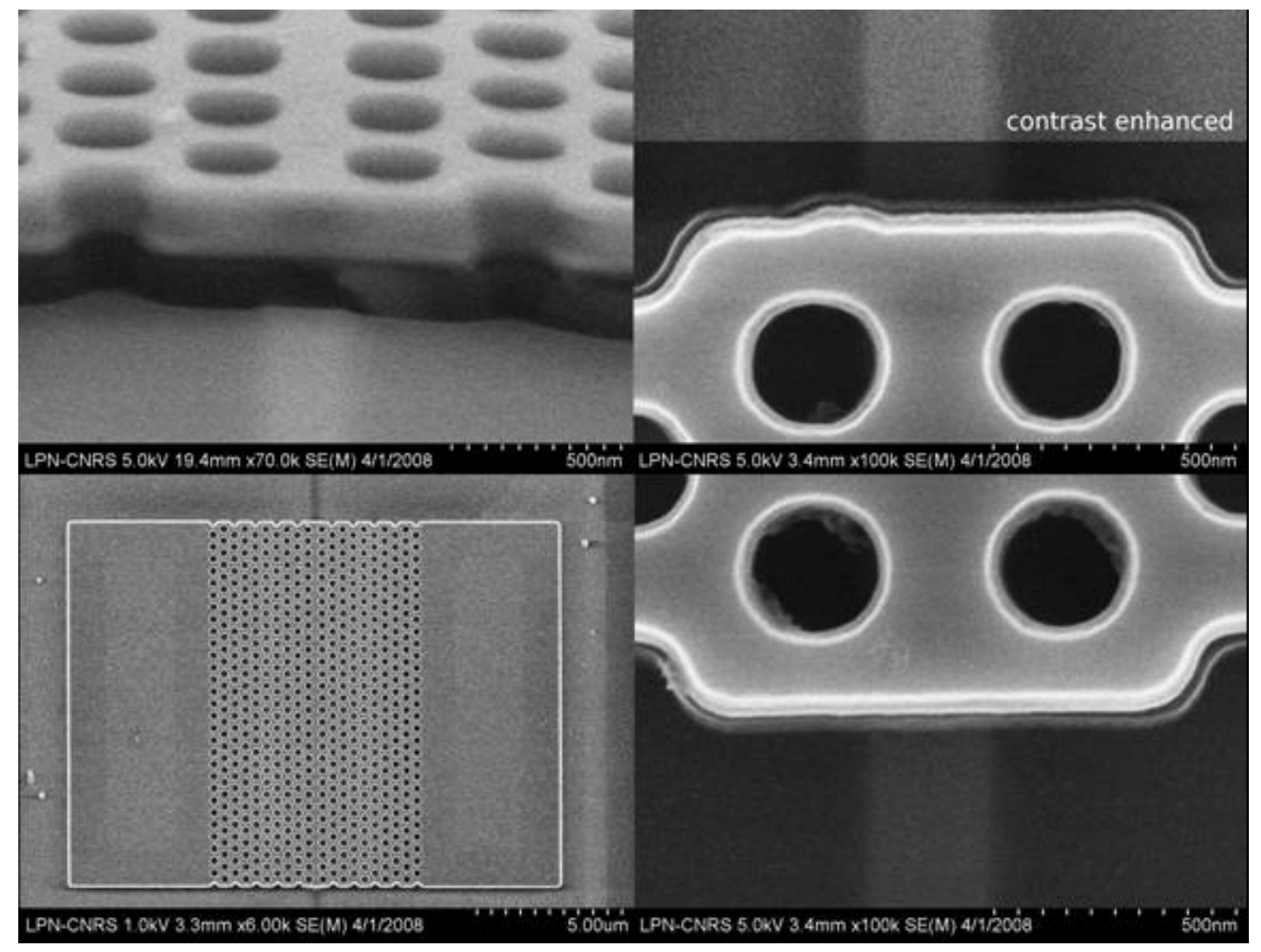

Figure 3.4: above left: angled view of end of sample showing patterned InP layer on top of etched $\mathrm{BCB}$, the Si guide is barely visible to the SEM, here we see a slightly lighter region,below left: full InP mesa containing etched PhC,above right: LHS of guide with enhanced contrast to display alignment with underlying waveguide below right: RHS of waveguide.

\subsubsection{Demonstration of optically pumped PhC lasers heterogeneously integrated and evanescently coupled to SOI}

The samples are surface pumped using a long working distance objective. 100fs pulses are generated at $810 \mathrm{~nm}$ by a Ti:Sa laser operating at a repetition rate of $80 \mathrm{MHz}$. This NIR pump is absorbed by all the semiconductor materials InP, quantum well and their barriers.

Then, the light emitted by the 2DPhC and coupled to the SOI wire, is collected at the output of the other grating by a SMF-28 optical fibre positioned at $10^{\circ}$ angle with respect to the sample surface in order to maximise collection at $1.55 \mu \mathrm{m}$. The emission is analysed using a spectrometer equipped with a cooled InGaAs CCD camera. Firstly, we observe the photoluminescence spectrum of the samples in order to identify the slow modes of interests by comparing the results with the modelling. The PL spectra of the samples for $\mathrm{a}=455 \mathrm{~nm}$ and 
$\mathrm{a}=445 \mathrm{~nm}$ show 3 clear peaks at the normalised frequencies corresponding to the low group velocity antisymmetric modes of the $\mathrm{W} 1$ waveguides. The band structure calculations help us to clearly identify the origin of the modes.

As the pump energy is increased, laser emission is observed at $1530 \mathrm{~nm}$ for the light emitted in the guide with a $=455 \mathrm{~nm}$. We plot on Figure 3.5 in log-log scale, the laser emission energy as a function of the pump energy coupled into the SOI waveguide. We can see that the curve is a "classical" S-shaped curve from which a threshold of about $5 \mathrm{pJ}$ is deduced. Near the threshold, the FWHM of the laser peak is $1 \mathrm{~nm}$. In this experiment, the maximum measured laser output pulse energy is of about $1.7 \mathrm{fJ}$ corresponding to an average power of $20 \mathrm{nW}$.

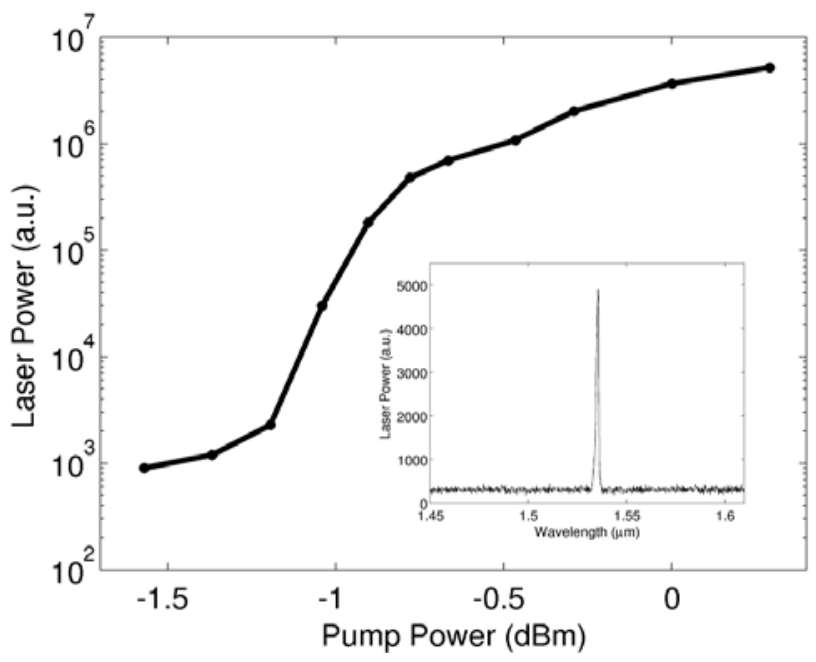

Figure 3.5: a) L-L curve for surface pumped emission, b) inset: laser spectrum at $1 \mathrm{~mW}$ pump power

\subsection{Electrically pumped III-V Photonic Crystal Lasers}

These lasers will be directly electrical pumped and all-optical flip-flop realization will be pursued with these devices. The membranes will be bonded on a CMOS-chip with the BCBtechnique of IMEC.

\subsubsection{Active passive structures}

The first activities involved a study to determine the layer stack for the active/passive structures. Trade-offs have been determined on optical, electrical and thermal aspects. Furthermore the basic technology is being developed by realizing passive structures as shown in Figure 3.6. A membrane ring resonator with a Q of 15000 has been realized in this way. In a second realization of passive membrane structures even better performances were obtained, with propagation loss of photonic wires below $7 \mathrm{~dB} / \mathrm{cm}$ and excess loss of only $0.6 \mathrm{~dB}$ for a 3dB MMI splitter.
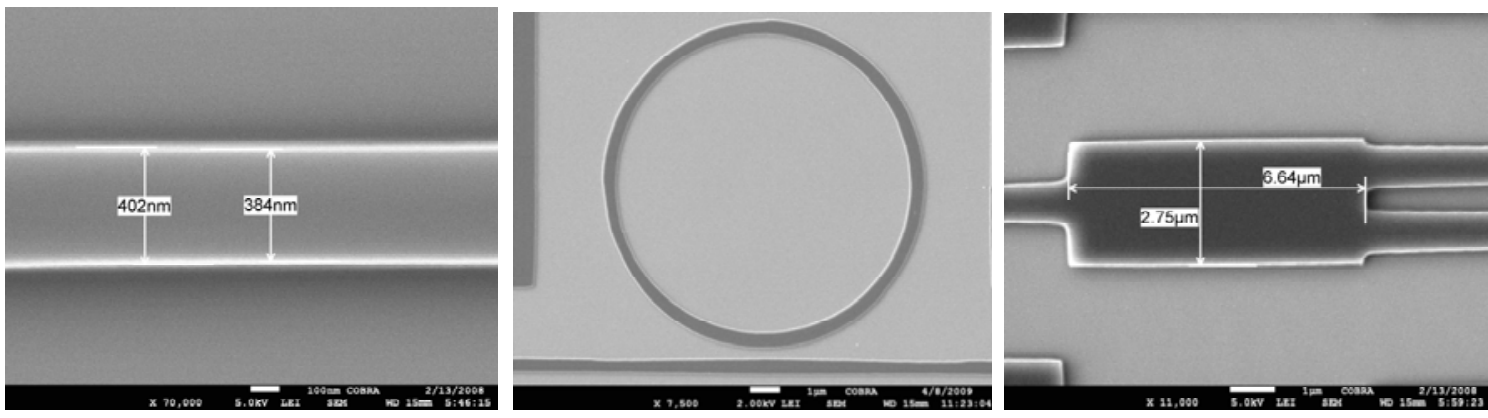
Fig. 3.6: a) 400 nm wide waveguide, b): $5 \mu$ m radius ring,

c): 3dB MMI splitter

In the second step, an investigation was started into the use of active-passive integration in membranes since most PICs consists of both active and passive components. In particular a test on the shape and orientation of the submicron size active regions has been done (in cooperation with Philips). The process development for this involved a "lift off" process to reduce the exposure time in EBL, which works quite well. Also a test was done for the optimum "wet etching time" in the active/passive butt joint regrowth processing. This was successful and the wet etching time now can be optimally chosen.
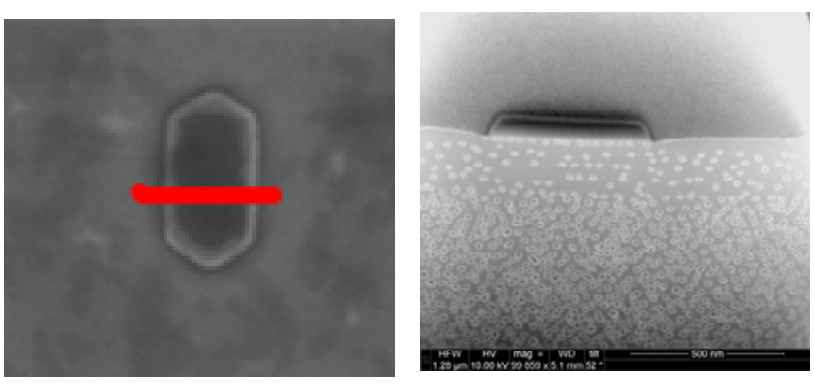

Fig.3.7:a) SEM picture, b): Cross section made by FIB

The butt-joint of active-passive interface was checked by FIB and the result looked quite good in terms of morphology. A smooth interface of the active and passive material is obtained as shown in Fig.3.7. Afterwards, micro photoluminescence from the small structures was measured(shown in Fig.3.8). The results shows that QWs of the small structures give emission at the designed wavelength range, and thus are alive optically. However, it is found that a size dependent blue shift of the PL spectrum appears, which points to quantum well intermixing effects. This is due to the defects caused in the dry etching process, which diffuse to the rest oft he active area during the high temperature regrowth process.
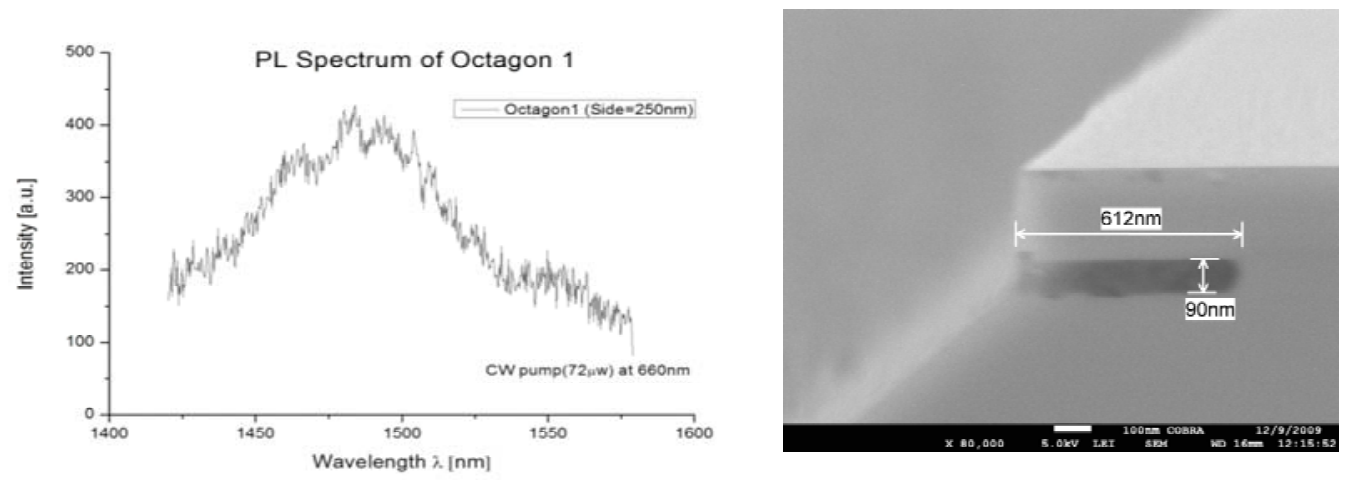

Fig. 3.8: PL spectrum of one submicron region

Fig. 3.9: Oxidized AlInAs in InP membrane

\subsubsection{Electrical pumping.}

An investigation has also started into the incorporation of current blocking layers in the membrane. To this end, in cooperation with Cedova, AlInP layers have been grown, which are expected to obtain a high resistivity after oxidation.

Finally, simulations have started into the design of photonic crystal lasers, with the aim to use these in a flip-flop configuration. 


\section{References:}

[1] S. Zhang, D. Owens, Y. Liu, M. Hill, D. Lenstra, A. Tzanakaki, G.-D. Khoe, H. Dorren, 'Multistate Optical Memory Based on Serially Interconnected Lasers', IEEE Phot. Techn. Lett., Vol. 17, pp. 1962-1964, Sept. 2005

[2] J. Wang, G. Berrettini, G. Meloni, L. Poti, A. Bogoni, 'All-Optical Clocked D-type FlipFlop Exploiting SOA-Based Optical SR Latch and Logic Gates‘, Proc. Photonics in Switching, pp. 151-152, Pisa, Sept. 2009.

[3] L. Gelens, S. Beri, G. Vandersande, J. Danckaert, 'Exploring Multistability in Semiconductor Ring Lasers: Theory and Experiment‘, Phys. Rev. Lett. 102, 193904, 2009.

[4] J. Van Campenhout, P. Rojo Romeo, P. Regreny, C. Seassal, D. Van Thourhout, S. Verstuyft, L. Di Cioccio, J. -M. Fedeli, C. Lagahe, and R. Baets, 'Electrically pumped InPbased microdisk lasers integrated with a nanophotonic silicon-on-insulator waveguide circuit', Opt. Express 15, 6744-6749, 2007.

[5] L. Liu, R. Kumar, K. Huybrechts, T. Spuessens, G. Roelkens, E-J. Geluk, T. de Vries, P. Regreny, D. Van Thourhout, R. Baets, G. Morthier, 'An ultra-small, low-power all-optical flip-flop memory on a silicon chip', Nature Photonics, Vol. 4, Nr. 3, pp. 182-187, March 2010.

[6] O. Raz, L. Liu, D. Van Thourhout, P. Rojo-Romeo, J.M. Fedeli, H.J.S. Dorren, 'High Speed Wavelength Conversion in a Heterogeneously Integrated Disc Laser Over Silicon On Insulator for Network on a Chip Applications', ECOC 2009 (paper Tuesday, session 4.2). [7] O. Raz, L. Liu, R. Kumar, G. Morthier, D. Van Thourhout, P. Regreny, P. Rojo-Romeo, T. De Vries, H.J.S. Dorren, 'Compact, Low Power and Low Threshold Electrically Pumped Micro Disc Lasers for 20Gb/s Non Return to Zero All Optical Wavelength Conversion’, paper OMQ5, OFC2010, San Diego, March 2010.

[8] R. Kumar, K. Huybrechts, L. Liu, T. Spuessens, G. Roelkens, E-J. Geluk, T. de Vries, P. Regreny, D. Van Thourhout, R. Baets, G. Morthier, 'An Ultra-small, Low-Power All-Optical Flip-Flop Memory on a Silicon Chip’, paper OTuN7, OFC2010, San Diego, March 2010.

[8] F. Raineri, A.M. Yacomotti, T.J. Karle, R. Hostein, R. Braive, A. Beveratos, I. Sanges, R. Raj, 'Dynamics of band-edge photonic crystal lasers', Optics Express, Vol. 17, pp. 31653172, March 2009.

[9] Y. Halioua, T. Karle, I Sagnes, G. Roelkens, D. Van Thourhout, R. Raj, F. Raineri, 'InP 2D Photonic Crystal Lasers integrated onto SOI waveguides‘, Proc. CLEO/QELS 2009, pp. 630-631, 2009.

[10] Y. Halioua, T. Karle, F. Raineri, P. Monnier, I Sagnes, G. Roelkens, D. Van Thourhout, R. Raj, F. Raineri, 'Hybrid InP-based photonic crystal lasers on silicon on insulator wires', Appl. Phys. Lett., Vol. 95, Nr. 20, Art. Nr. 201119, Nov. 2009 